\title{
Polysémie et polytaxie verbales entre synchronie et diachronie
}

\author{
Jacques FRANÇOIS \\ Université de Caen \& CRISCO, EA 4255 \\ jacques.francois@unicaen.fr
}

\section{Introduction}

La corrélation dominante entre variation sémantique et variation syntaxique est largement admise, même s'il ne s'agit que d'une tendance. F. Rastier (1991:139) affirme que "des composants sémantiques déterminent des valences syntaxiques », R. van Valin (2005) considère - contrairement à Jackendoff $(1990,2002)$ - que la corrélation est guidée par le sémantisme du verbe, les Grammaires de Construction (spécialement Goldberg 1995 pour le lexique verbal) décrivent le sémantisme propre de constructions verbales indépendamment des lexèmes verbaux auxquels elles s'associent et Dubois \& Dubois-Charlier (1997a) regroupent 25610 entrées verbales en 14 classes génériques essentiellement sur la base d'une analyse distributionnelle et transformationnelle.

D'un point de vue diachronique, je propose de forger la notion de «néologie syntaxique » sur le modèle de la «néologie sémantique » proposée par L. Guilbert (1975) pour rendre compte de la diversification progressive des sens. La néologie syntaxique concerne la diversification progressive des cadres prédicatifs ${ }^{i}$, laquelle est presque toujours associée à une diversification sémantique. Si le produit de la néologie sémantique est appelé « polysémie » depuis Bréal (1897/2005), celui de la néologie syntaxique peut être appelé "polytaxie»- à l'exception des cadres prédicatifs qui entretiennent des relations transformationnelles au sens classique de Z. Harris et M. Gross et constituent ce que W. Busse appelle une Produktklasse (classe pluriforme, 1974) et Dubois \& Dubois-Charlier (1997a) un schème syntaxique composé.

Le propos de ce «point de vue » est, après avoir rappelé les grandes orientations de l'analyse de la polysémie des verbes français $(\S 1)$, de clarifier l'articulation entre la polysémie et la polytaxie $(\S 2)$ en comparant le positionnement relatif des entrées verbales dans des hiérarchies conceptuelles (Stein 2005, Dictionnaire Intégral de la société Mémodata), la composition de la base de données lexicale Les verbes français (Dubois \& Dubois-Charlier 1997) et la méthode de cartographie de la polysémie verbale à partir de liens intersynonymiques (François 2007a, 2008b). Le $\$ 3$ est enfin consacré à l'esquisse d'une approche diachronique de la polysémie verbale à partir des travaux de P. Koch et d'observations sur des néologies syntaxiques frappantes (François 2007b, 2008a). Les classes distributionnelles (et transformationnelles dans la conception de Dubois \& Dubois-Charlier 1997) ont certainement une source sémantique, mais cette source ne peut pas rendre compte du profil sémantaxique de chaque verbe et la perte de prédicativité, c'est-à-dire la dégradation du lexème verbal à un statut d'auxiliaire, de verbe support de prédication nominale ou de tête d'une locution verbale (cf. François à par.), est associée à une désémantisation dont un effet secondaire à examiner soigneusement peut être le développement de structures lexico-grammaticales sans 'armature' sémantique.

\section{Les orientations dominantes de l'analyse de la polysémie verbale en français}

Je distinguerai cinq approches principales de la polysémie verbale, la première relevant dans la terminologie de Victorri \& Fuchs (1996, chapitre 2) de la polysémie « horizontale », la seconde occupant une position intermédiaire, et les trois suivantes souscrivant à une conception «verticale» de la polysémie. 
1.1. La conception «horizontale » de la polysémie est typique de la sémantique cognitive de la côte ouest des USA. C'est ainsi par exemple que Fillmore \& Atkins (2000) étudient la différenciation des sens des verbes anglais crawl et français ramper à partir d'un noyau prototypique commun et d'extensions de sens partiellement divergentes. Le noyau comporte deux branches : mouvement naturel d'espèces animales et mouvement des humains délibéré ou causé par l'épuisement. A partir de ce noyau, crawl connaît une extension de sens appliquée au pullulement des insectes, tandis que ramper en connaît une autre relative à l'expansion d'une entité appropriée, par exemple le feu ou les ténèbres.

1.2. Les conceptions de R. Martin et de F. Rastier se situent dans le cadre du structuralisme sémantique, opérant à partir de la notion de sémème prédicatif une distinction de base pour les prédicats entre polysémie interne et externe (Martin 1983) et plus précisément entre quatre types de variation contextuelle du sémème (Rastier 1991).

R. Martin (1983:74-83) introduit trois niveaux principaux de classement (cf. François 1989 :541-4). L'homonymie se distingue de la polysémie par l'absence de tout sème commun. La polysémie interne " concerne le sémème» tandis que la polysémie externe "effecte limitativement les actants ». La polysémie interne présente deux sous-classes : celle d'acceptions « suppose soustraction ou addition d'un ou plusieurs sèmes (ex. grelotter, par métonymie « se sentir transi »), tandis que celle de sens résulte de la « combinaison de la soustraction et de l'addition de sèmes » (ex. couler 1 «se déplacer (liquide)» vs. couler 2 《aller au fond de l'eau»). De son côté, la polysémie externe présente trois sous-classes: d'acceptions (par ex. cambrioler qn dérivant par métonymie de cambrioler un local), de sens (ex. apprendre la danse vs. une nouvelle à qn) et sélectionnelle, liée à une "répartition différente des fonctions grammaticales sur un même schème profond de nature sémantico-logique » (ex. qn apprend la danse (avec qn) vs. qn apprend la danse à qn).

F. Rastier (1991:150) spécifie quatre types de polysémie « interne » selon Martin, mais il ne reconnaît ce statut qu'au premier type : (i) non-instanciation d'un ou de plusieurs attributs (sans correspondance chez Martin) ; (ii) création d'attributs (chez Martin : polysémie interne d'acceptions par addition de sèmes) ; (iii) délétion d'attributs (chez Martin : polysémie interne d'acceptions par soustraction de sèmes); (iv) création d'attributs, combinée à la délétion d'autres attributs (chez Martin : polysémie interne de sens).

1.3. L'analyse psychomécanique, représentée par les travaux de J. Picoche ${ }^{\mathrm{ii}}$, se fonde sur trois notions : le CINETISME est défini comme une « sorte de trajectoire sémantique dont tout point peut, en principe être le siège d'une immobilisation par le discours" $(1986: 7)$ et qui progresse "selon une chronologie de raison» de l'universel vers le particulier, différentes SAISIES se rangeant au long du continuum du cinétisme, de la saisie précoce (proche du début du cinétisme) à la saisie tardive (proche de son aboutissement) et enfin à la saisie plénière (au terme du cinétisme) et l'opération de SUBDUCTION correspond à un parcours du continuum du sens en langue (la source du cinétisme) aux effets de sens en discours (son aboutissement). J. Picoche distingue explicitement la chronologie « de raison » relative à la dérivation cognitive des types de procès évoqués par le verbe et la chronologie effective des diversifications de sens $\mathrm{s}^{\mathrm{iii}}$. Mais existe-t-il effectivement une "rationalité » du parcours sémantique, comme le suppose l'approche de J. Picoche ? L'ouvrage récent de Rakova, Pethö \& Rákosi (2007) sur la base cognitive de la polysémie, c'est-à-dire concrètement sur « la réalité psychologique de la polysémie linguistique ${ }^{\text {iv }}$ fournit des données expérimentales pour y voir plus clair.

1.4. Comme la notion de SIGNIFIE DE PUISSANCE de J. Picoche, celle de FORME SCHEMATIQUE définie par Franckel, Paillard \& Saunier (1997 : 51) comme « un dispositif abstrait jouant un rôle de régulation d'une variation qu'elle permet d'articuler sur plusieurs plans » illustre la préférence pour une conception « verticale » de la polysémie. La forme schématique associée à passer est formulée ainsi : "étant donnée une discontinuité sur une continuité première, passer reformule cette discontinuité comme continuité ». Les auteurs précisent immédiatement que la continuité première peut être spécifiée comme une trajectoire, un mouvement, un écoulement ou plus généralement un «état de référence » et la discontinuité comme un obstacle, un point d'observation, un hiatus ou une hétérogénéité. Cette approche représente une idéalisation des processus cognitifs de mise en réseau des différentes acceptions du verbe 
passer, dont on ne peut pas savoir, en dehors d'une démarche de psycholinguistique expérimentale, s'ils ont une réalité mentale.

1.5. Enfin l'analyse en «schèmes sémantico-cognitifs » privilégiée par J.P. Desclés (cf. 2005 à propos du verbe avancer) semble mieux répondre au critère de réfutabilité parce que chaque schème constitue « un agencement, au moyen d'opérations précises (de spécification, emboîtement et abstraction), de primitives sémantico-cognitives de perception et d'action ».

Dans son chapitre 1 consacré à un inventaire des conceptions actuelles de la polysémie lexicale, Stein (2005:16-21) consacre en outre une section à l'apport du « lexique génératif » de J. Pustejovsky (1995)n lequel est certes une orientation importante de la sémantique lexicale qui a donné matière à débat en France (cf. Kleiber 1999), mais qui n'a pas à ma connaissance donné lieu à des applications majeures en linguistique française.

\section{L'articulation entre la polysémie et la polytaxie}

\subsection{La hiérarchisation conceptuelle au service de la désambiguïsation automatique de la polysémie verbale (Stein 2005, Dictionnaire Intégral Memodata)}

Achim Stein (2005) défend l'idée que la hiérarchisation fine des types de concepts prédicatifs permet,

i. non seulement un classement onomasiologique des différents sens d'un verbe, présentés (comme dans EuroWordNet, mais avec une démarche sémantiquement et syntaxiquement plus élaborée qui s'apparente à celle de Dubois \& Dubois-Charlier, 1997, pour les verbes français) comme des entrées lexicales sans attachement d'un sens de base à l'une d'entre elles,

ii. mais surtout le calcul de la distance sémantique entre ces prédications, calcul pondéré par la prise en compte de liens transversaux.

A titre d'illustration, l'auteur présente p. 92 le diagramme hiérarchique ${ }^{v}$ de la spécification des 10 entrées du verbe italien ricambiare.La procédure d'attachement de ces 10 entrées lexicales à l'arbre de hiérarchisation permet d'évaluer la distance sémantique entre les entrées (évaluation pondérable par la prise en compte de liaisons transversales touchant en particulier les variantes causative et non causative d'un même concept prédicatif). Ainsi ricambia 3 et 7 et ricambia 8 et 9 , qui sont attachés à un même nœud, respectivement «RemplacerQc » et «ChangerVestim» sont les entrées les plus étroitement apparentées. Ces quatre entrées entretiennent une relation un peu moins étroite avec ricambia 6 attaché au nœud EchangerContre, étant donné que les trois noeux RemplacerQc, EchangerContre et ChangerVestim sont directement dominés par le même nœud Remplacer. Leur relation avec ricambia 4 et ricambia 5 est quasiment inexistante, car il faut remonter du $7^{\mathrm{e}}$ au $2^{\mathrm{e}}$ niveau de hiérarchisation conceptuelle pour trouver un nœud dominant commun «Affecter». A l'aide d'une telle procédure, on peut reconstruire par éloignement progressif la perte de «ressemblance de famille» qui est à la source de l'intuition d'homonymie. Cette intuition émerge progressivement en fonction du nombre de nœuds à franchir pour interconnecter deux entrées. J'ai cherché sur la figure 1 à rendre compte visuellement de la proximité entre les entrées par des cadres de plus en plus discontinus. 
Niveau de hiérarchie conceptuelle

entrée lexicale traduction

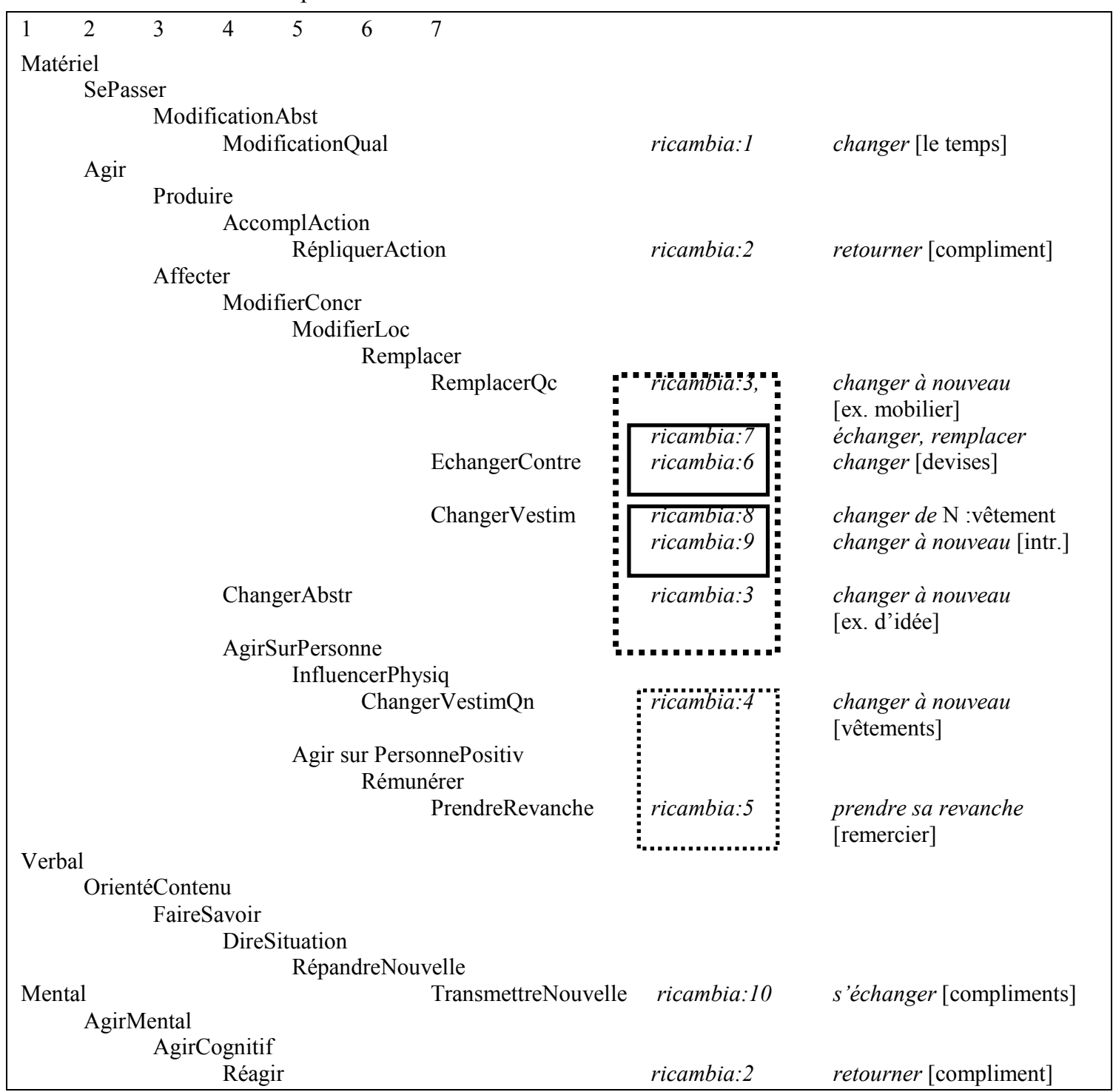

Figure 1 : La polysémie du verbe italien ricambiare représentée par attachement à des noeuds conceptuels hiérarchisés

Le Dictionnaire intégral de la société Mémodata (édité sous le nom DICOLOGIQUE) propose un classement dans le même esprit, mais qui concerne l'ensemble du lexique français. A titre d'illustration (cf. Figure 3) le verbe changer est rattaché en tant que «v.tr.intr. » (rubrique 1) à une liste de concepts dont la tête est CHANGER (8178 verbes). Parmi les sous-concepts de rang 2 et 3 dominés par CHANGER (cf. Figure 2), les types d'emploi de la rubrique 1 relèvent de : 


\begin{tabular}{|lll|}
\hline concept rang 1 & concept rang 2 & concept rang 3 \\
CHANGER & $\rightarrow$ DEVENIR - FAIRE DEVENIR & $\rightarrow$ FAIRE DEVENIR AUTRE \\
& $\rightarrow$ AUGMENTER - DIMINUER & $\rightarrow$ DEVENIR AUTRE \\
$\rightarrow$ DÉTÉRIORER & $\rightarrow$ DIMINUERTER \\
& $\rightarrow$ DÉTRUIRE \\
& $\rightarrow$ NUIRE (À QQN) \\
& $\rightarrow$ SE DETERIORER \\
& $\rightarrow$ ALTERER \\
& $\rightarrow$ DEVENIR PIRE \\
& $\rightarrow$ USER \\
& $\rightarrow$ DEFORMER ALTERER LA FORME DE QQCH \\
& $($ ETC.)
\end{tabular}

Figure 2 : La hiérarchie conceptuelle associée à CHANGER

La figure 3 reproduit l'arbre de hiérarchisation de DICOLOGIQUE pour le verbe changer. Seuls les quatre premiers nœuds de la rubrique 1 « changer, v.tr.intr. » ont été détaillés pour faire apparaître

les concepts de rang 3. Pour une comparaison entre l'organisation de la polysémie du verbe changer dans le Dictionnaire intégral et dans l'actuel WordNet pour le français, on peut consulter Dutoit \& François (2007, Annexe, p.57). 


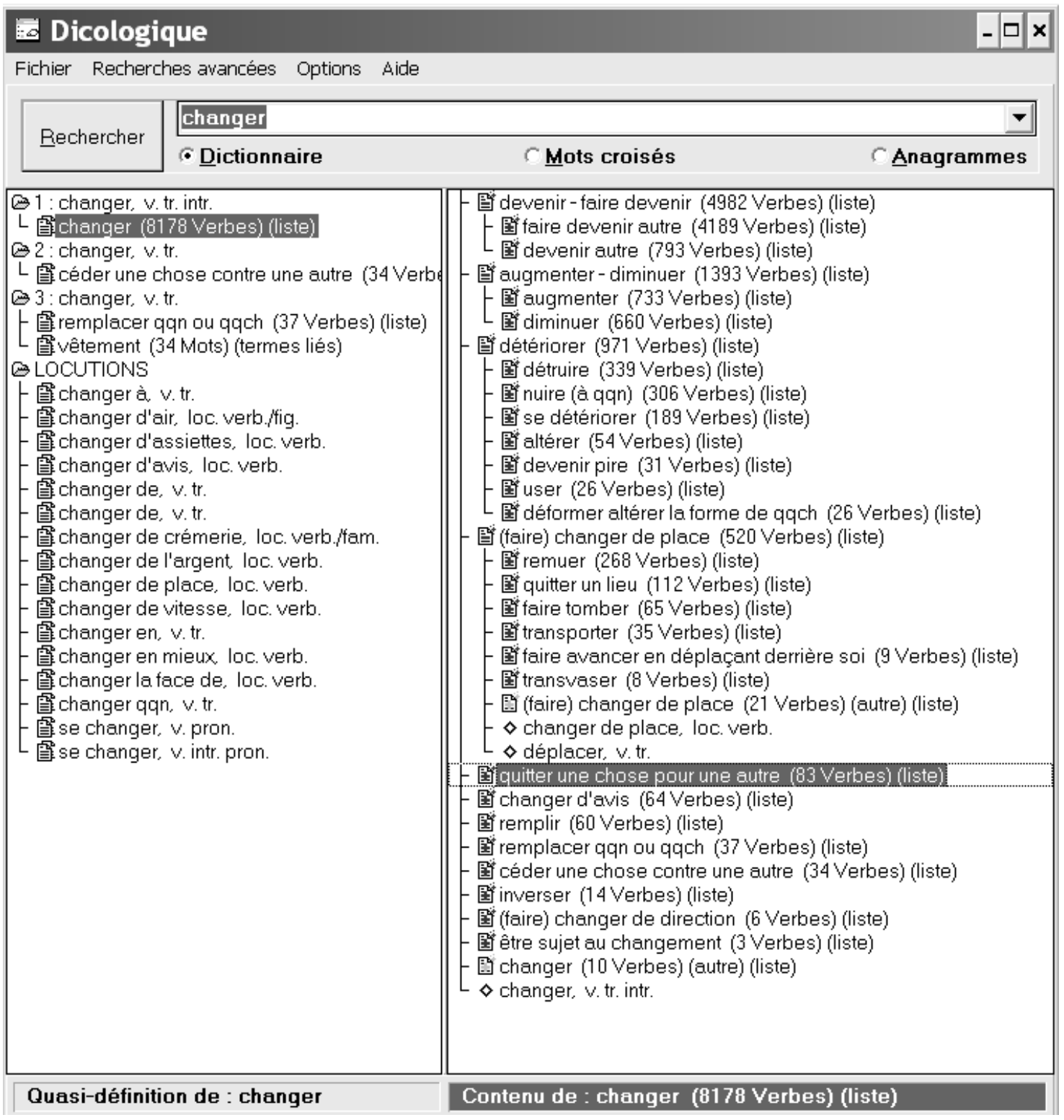

Figure 3 : Représentation partielle de l'attachement des entrées du verbe français changer à des nœuds conceptuels hiérarchisés selon DICOLOGIQUE (Dictionnaire intégral de la société Mémodata) 


\subsection{De l'analyse distributionnelle à la décomposition sémantique : Les Verbes français (Dubois \& Dubois-Charlier 1997a, 1997b)}

Profitant de la longue expérience lexicographique ${ }^{\mathrm{vi}}$ de Jean Dubois, les auteurs ont mené à bien avec Les verbes français une base de données syntactico-sémantique exhaustive du lexique verbal français " formée de 25610 entrées verbales représentant 12310 verbes différents, dont 4188 à plusieurs entrées » (Dubois \& Dubois-Charlier 1997a, p.1). L'édition papier de 1997 présente un classement de ces 25610 entrées verbales dans 14 classes génériques: «communication, mouvement avec ou sans locatif, transformation, don et privation, saisie et possession, mise en relation, état psychique ou comportement social, auxiliaires » (ibid. p.3).

De ce fait, la première impression en consultant cette édition est que la démarche est 'descendante' (dans la terminologie de la psychologie cognitive), c'est-à-dire va d'un classement conceptuel a priori vers des réalisations morphosyntaxiques. En réalité, la démarche est inverse, comme l'expliquent les auteurs dans leur article de la même année (1997b), à la base l'entreprise est distributionnelle et transformationnelle : le point de départ est le repérage des constructions A, N, P, T (respectivement : intr., tr.ind., pron. et tr.), puis le regroupement des schèmes de constructions composés (par exemple $\mathrm{T} P$ pour les entrées primairement tr. et secondairement pron. vs. P T pour les entrées primairement pron. et secondairement tr., cf. François 2008c).

Sur cette base les auteurs établissent la liste des entrées verbales (de 1 pour 4188 verbes et jusqu'à 61 pour le verbe passer) et associent à chacune un descriptif sémantique intitulé Opérateur. Par une prise en compte parallèle de la morphosyntaxe (la rubrique Constructions) et de la sémantique (la rubrique Opérateur), 54 classes syntactico-sémantiques sont progressivement distinguées, elles-même regroupées dans les 14 classes génériques mentionnées plus haut.

L'édition papier ne mettait pas en évidence l'exploitabilité de la base de donnnées pour l'étude de la polysémie verbale, mais l'édition électronique désormais disponible ${ }^{\mathrm{vii}}$ favorise cette approche. Dans le prolongement des illustrations des bases de données de Stein et de la société Mémodata, nous reproduisons ci-dessous les 19 entrées $^{\text {viii }}$ du verbe changer $^{\text {ix }}$. 


\begin{tabular}{|c|c|c|c|c|c|c|}
\hline Entrée & Dom & $\operatorname{Css}^{\mathrm{x}}$ & Opérateur & Paraphrase & Exemples $^{\mathrm{xi}}$ & Constr $^{\text {xii }}$ \\
\hline changer 01 & $\mathrm{COM}$ & T3f & $\begin{array}{l}\text { li.mut } \\
\text { CT/PR } \\
\end{array}$ & échanger contre & $\begin{array}{l}\text { On c } \sim \text { des livres pour des disques, } \\
\text { une place pour une autre. } \\
\end{array}$ & $\mathrm{T} 13 \mathrm{k} 0$ \\
\hline changer 02 & $\mathrm{MON}$ & T3d & $\begin{array}{l}\text { mut } \\
\text { monnaie E } \\
\text { autre }\end{array}$ & convertir & $\begin{array}{l}\text { On c } \sim \text { des dollars en francs. } \\
\text { Cette monnaie ne se c } \sim \text { pas. }\end{array}$ & $\begin{array}{l}\text { T13j0 } \\
\text { P30j0 }\end{array}$ \\
\hline changer 03 & OBJ & T3f & $\begin{array}{l}\text { li.mut D } \\
\text { obj }\end{array}$ & remplacer & On c $\sim$ d'assiettes. & $\mathrm{N} 1 \mathrm{~b}$ \\
\hline changer 04 & LOC & $\mathrm{T} 2 \mathrm{e}$ & $\begin{array}{l}\text { li.mut D } \\
\text { place av qn }\end{array}$ & $\begin{array}{l}\text { replacer par } \\
\text { qc,qn }\end{array}$ & On c $\sim$ sa voiture,les invités de place. & T19b0 \\
\hline changer 05 & SOC & $\mathrm{T} 4 \mathrm{e}$ & \begin{tabular}{|l|} 
li.mut \\
CT/PR \\
\end{tabular} & troquer & $\begin{array}{l}\text { On c un métier avec, } \\
\text { pour une autre activité. }\end{array}$ & $\mathrm{T} 13 \mathrm{k} 0$ \\
\hline changer 06 & LIT & R4c & $\begin{array}{l}\text { m.e.état } \\
\text { abs }\end{array}$ & modifier & On c $\sim$ le sens avec une virgule. & $\begin{array}{l}\text { T1306 } \\
\text { P3000 }\end{array}$ \\
\hline changer 07 & HAB & T3f & $\begin{array}{l}\text { li.mut D } \\
\text { hab }\end{array}$ & $\begin{array}{l}\text { s'habiller } \\
\text { autrement }\end{array}$ & On c $\sim$ de vêtements. & $\mathrm{N} 1 \mathrm{~b}$ \\
\hline changer 08 & PSY & E2b & $\begin{array}{l}\text { f.ex qn D } \\
\text { abs }\end{array}$ & distraire & $\begin{array}{l}\text { Ceci c } \sim \mathrm{P} \text { de ses ennuis. } \\
\text { On se } \mathrm{c} \sim \text { de ses soucis en sortant. }\end{array}$ & $\begin{array}{l}\text { T31b0 } \\
\text { P10b0 }\end{array}$ \\
\hline changer 09 & $\mathrm{COM}$ & T3f & \begin{tabular}{|l|} 
li.mut AV \\
qc \\
\end{tabular} & $\begin{array}{l}\begin{array}{l}\text { remplacer qc } \\
\text { contre }\end{array} \\
\end{array}$ & $\begin{array}{l}\text { On c } \sim \text { les perles du collier pour des } \\
\text { diamants. }\end{array}$ & $\begin{array}{l}\mathrm{T} 13 \mathrm{c} 0 \\
\mathrm{P} 8000 \\
\end{array}$ \\
\hline changer 10 & SOC & $\mathrm{T} 2 \mathrm{e}$ & $\begin{array}{l}\text { li.mut D } \\
\text { abs }\end{array}$ & $\begin{array}{l}\text { passer de qc à qc } \\
\text { autre }\end{array}$ & $\begin{array}{l}\text { On c } \sim \text { de poste,d'orientation } \\
\text { dans ses études. }\end{array}$ & N1b \\
\hline changer 11 & VEH & T3f & $\begin{array}{l}\text { li.mut D } \\
\text { véh }\end{array}$ & $\begin{array}{l}\text { prendre autre } \\
\text { transport }\end{array}$ & $\begin{array}{l}\text { On c } \sim \text { de ligne à la station Châtelet. } \\
\text { On c } \sim \text { de train,d'avion. }\end{array}$ & $\mathrm{N} 1 \mathrm{~b}$ \\
\hline changer $12(\mathrm{~s})$ & PSY & $\mathrm{T} 2 \mathrm{a}$ & \begin{tabular}{|l|} 
mut qn E \\
autre
\end{tabular} & transformer & $\begin{array}{l}\text { La prison a c } \sim \mathrm{P} \text { en un monstre. } \\
\text { Cet homme se c } \sim \text { en démon. }\end{array}$ & $\begin{array}{l}\mathrm{P} 10 \mathrm{j} 0 \\
\mathrm{~T} 31 \mathrm{j} 0 \\
\end{array}$ \\
\hline changer 13 & TPS & $\mathrm{T} 4 \mathrm{~d}$ & \begin{tabular}{|l|} 
(qc)mut E \\
autre
\end{tabular} & évoluer & $\begin{array}{l}\text { La ville } \mathrm{c} \sim \text { d'aspect. } \\
\text { Le pays } \mathrm{c} \sim \text { beaucoup. }\end{array}$ & A30 \\
\hline changer 14 & SOM & $\mathrm{T} 4 \mathrm{~d}$ & \begin{tabular}{|l|} 
(qc)mut d \\
aspect
\end{tabular} & muer & La voix c $\sim$ & $\mathrm{A} 30$ \\
\hline changer 15 & PSY & $\mathrm{T} 2 \mathrm{a}$ & $\begin{array}{l}\text { (qn)mut } \\
\text { abs } \\
\end{array}$ & évoluer,varier & On c $\sim$ avec le temps. & A16 \\
\hline changer 16 & SOC & $\mathrm{T} 2 \mathrm{e}$ & li.mut D qn & $\begin{array}{l}\text { remplacer p qn } \\
\text { d'autre }\end{array}$ & On $\mathrm{c} \sim$ de directeur. & $\mathrm{N} 1 \mathrm{~b}$ \\
\hline changer 17 & SOC & $\mathrm{T} 2 \mathrm{e}$ & $\begin{array}{l}\text { li.mut D } \\
\text { situa av qn }\end{array}$ & $\begin{array}{l}\text { modifier } \\
\text { affectation de }\end{array}$ & On c $\sim \mathrm{P}$ de poste. & T19b0 \\
\hline changer $18(\mathrm{~s})$ & HAB & N1a & \begin{tabular}{|l|}
$\begin{array}{l}\text { mun soi D } \\
\text { hab-nv }\end{array}$ \\
\end{tabular} & $\begin{array}{l}\text { changer de } \\
\text { vêtements }\end{array}$ & $\begin{array}{l}\text { On se c } \sim \text { pour sortir. } \\
\text { On c } \sim \text { un enfant de vetements. }\end{array}$ & $\begin{array}{l}\text { P10b0 } \\
\text { T11b0 } \\
\end{array}$ \\
\hline changer 19(s) & MTO & $\mathrm{T} 3 \mathrm{~b}$ & \begin{tabular}{|l|} 
(qc)mut soi \\
E autre
\end{tabular} & se résoudre en & La neige se c $\sim$ en pluie. & P30j0 \\
\hline
\end{tabular}

Tableau 1 : Descriptif des 19 entrées verbales du verbe changer dans l'édition électronique des VERBES FRANÇAIS

En allant au-delà du mode d'exploitation de la base de données que présentent les auteurs, on peut, sur la base de la décomposition et de la comparaison des Opérateurs, effectuer des regroupements entre les entrées comparables à ceux qu'effectue Stein (2005) à partir du calcul de proximité sur l'arbre de hiérarchisation conceptuelle. Ainsi l'Opérateur sémantique de 18 des 21 entrées du verbe changer comporte la composante « mut » désignant une mutation sous diverses formes :

i. 《mut » + entité $+\mathrm{E}(\rightarrow$ en $)+$ autre pour changer 02 et $\mathbf{1 2}(\mathbf{s})$

ii. «(qc)mut» (sujet qc de la construction intr.) pour changer 13, 14 et 19(s), «(qn)mut » (sujet qn de la construction intr.) pour changer 15.

iii. « li.mut » (mutation liée $\rightarrow$ échange $)+\mathrm{AV}(\rightarrow$ avec $)$ qc pour changer 09 ou $+\mathrm{CT} / \mathrm{PR}(\rightarrow$ contre, pour) pour changer $01,05$.

iv. «li.mut » $+\mathrm{D}(\rightarrow$ de $)+$ entité pour la majorité des entrées : changer 03, 04, 07, 10, 11, 16 et 17. 
Sur la base de la décomposition de l'Opérateur sémantique, on peut donc dégager les regroupements sémantiques suivants (Tableau 2) :

\begin{tabular}{|l|l|c|c|c|c|c|c|}
\hline \multicolumn{3}{|c|}{ mut } & \multicolumn{2}{c|}{ li.mut } & \multirow{2}{*}{ m.e.état } & f.ex & mun \\
\hline mut & (qc)mut & (qn)mut & $\begin{array}{l}\text { li.mut }+ \text { AV / } \\
+\mathrm{CT} / \mathrm{PR}\end{array}$ & li.mut $+\mathrm{D}$ & m & & \\
\hline $02,12(\mathrm{~s})$ & $13,14,19(\mathrm{~s})$ & 15 & $01,05,09$ & $\begin{array}{c}03,04,07, \\
10,11,16,17\end{array}$ & 06 & 08 & $18(\mathrm{~s})$ \\
\hline
\end{tabular}

Tableau 2 : Regroupement des 19 entrées du verbe changer à partir de la comparaison des composantes de la rubrique Opérateur

Pour conclure la comparaison entre les trois entreprises de Stein (2005), de la société Mémodata et de Dubois \& Dubois-Charlier, il est à noter que toutes trois partagent un principe essentiel : dans les trois cas

i. une analyse sémantique et syntaxique minutieuse est effectuée en premier ;

ii. une entrée lexicale est distinguée pour chaque configuration de propriétés sémantiques et syntaxiques ;

iii. les entrées ainsi constituées sont on peuvent être comparées et ordonnées par proximité décroissante, sans établissement a priori d'un critère de délimitation entre polysémie et homonymie.

\subsection{La cartographie de la polysémie verbale et le repérage des aires sémantaxiques (François 2007a, 2008b)}

Le Dictionnaire Électronique des Synonymes (DES) du CRISCO offre une troisième approche de la polysémie, de type «émergentiste » (cf. Ploux \& Victorri 1998). Au lieu d'être calculée sur la base du rattachement à des noeux conceptuels hiérarchisés comme à l'aide du programme Italian Verbs de Stein (2005) ou de la base conceptuelle du Dictionnaire intégral de Mémodata ou de la discrimination des entrées verbales à partir des schèmes de constructions composés et de l'attribution d'un Opérateur sémantique componentiel comme dans Les verbes français, l'exploitation du DES pour l'examen de la polysémie lexicale est basée sur les liens synonymiques fournis par 7 dictionnaires source du français $(2$ dictionnaires extensifs de langue, 2 dictionnaires des synonymes du $19^{\mathrm{e}}$ siècle et 3 dictionnaires des synonymes du $20^{\mathrm{e}}$ siècle). Une fois symétrisés, ces 450000 liens concernant 45000 items constituent un vaste réseau relationnel. Pour tout item examiné, le DES délivre un espace sémantique multidimensionnel (comportant autant de dimensions que de synonymes) matérialisé par un nuage de points. Ces points représentent les cliques de synonymes, c'est-à-dire les plus petits points d'intersection entre les espaces sémantiques des synonymes en cause. L'espace sémantique de l'item examiné est une représentaiton géométrique plane par défaut offrant la meilleure distribution possible du nuage des cliques.

A titre d'illustration, pour le verbe affecter, ses 4 principaux synonymes (c'est-à-dire ceux qui figurent dans le plus grand nombre de cliques de synonymes) sont localisés (cf. Figure 4)

i. $\quad$ dans la région médiane gauche pour altérer $(\Leftrightarrow q c$ affecte qc) et toucher $(\Leftrightarrow q c$ affecte qn)

ii dans la région supérieure droite pour nommer $(\Leftrightarrow q n$ affecte qn (à qn/àqc/qp))

iii dans la région inférieure droite pour destiner ( $\Leftrightarrow$ qn affecte $q c$ (à qn/à qc/qp))

Les cliques de synonymes compatibles avec les 4 constructions majeures du verbe affecter sont respectivement :

i. qc affecte qc, SYN : altérer

Une seule clique est associée à cette construction, à savoir : 2 \{affaiblir, affecter, altérer, atteindre. Cette clique inclut atteindre qui figure dans 4 cliques sur les 13 associées à la construction qc affecte qn. De ce 
fait il y a une seule aire sémantaxique pour $q n / q c$ affecte $q n$, la clique 2 constituant à elle seule la coordonnée de sens de la variante $q$ c affecte qn.

\section{ii. $\quad$ qc affecte qn, SYN : toucher}

L'aire sémantaxique (cf. figure 3) de qc affecte qn, localisée dans la région médiane gauche de l'espace sémantique du verbe sur le plan 1x2 par défaut est constituée de 13 cliques, ex. 10 \{ affecter, affliger, atteindre, frapper, toucher, troubler, émouvoir\}.Mentionné dans 7 cliques, le synonyme toucher joue un rôle central, mais son espace n'est pas exactement corrélé à celui de la construction qn affecte qn, car d'une part sa clique extrême à l'extrémité inférieure droite \{affecter, attaquer, jouer toucher\} comporte le synonyme jouer, lequel ne peut pas remplacer affecter dans la construction qc affecte qn (! qc joue qn) et d'autre part 3 des 13 cliques se situent en dehors de l'espace de toucher au-delà de son extrémité gauche.

\section{iii. $\quad$ qn affecte qn (à qn/à qc/qp), SYN : nommer}

Le troisième type d'emploi est représenté par qn affecte qn (à qn/à qc/qp). 7 cliques de synonymes, ex. 50 \{affecter, choisir, désigner, nommer, prendre\}, sont composées de synonymes partageant cette construction dans le même sens. Ici, le synonyme le plus mentionné (dans 5 des 7 cliques) est nommer, suivi de désigner (dans 3 cliques). L'aire sémantaxique ne couvre pas l'extrémité supérieure de l'espace de nommer parce que la clique \{affecter, désigner, nommer, révéler\} à l'extrémité supérieure de cet espace, ainsi que la clique $\{$ affecter, assigner, désigner, marquer $\}$, comportent un synonyme exclu, révéler pour l'une et marquer pour l'autre.

iv. $\quad$ qn affecte qc (à qc/à qn/qqp), SYN : attribuer / destiner

Enfin le dernier type d'emploi est celui de qn affecte $q c$ (à qc/à qn/qqp), représenté par 7 cliques de synonymes, ex. 24 affecter, appliquer, attribuer, consacrer, destiner \}. Le synonyme le plus mentionné est attribuer (figurant dans les 7 cliques), suivi de destiner (dans 3 cliques). Appliquer occupe la partie inférieure de l'aire sémantaxique, mettre sa partie gauche, classer la partie supérieure et destiner la partie droite $^{\text {xiii }}$..

La différence majeure entre la philosophie 'émergentiste' du mode de calcul des interdistances entre synonymes d'un même item que fournit ce dictionnaire ${ }^{\text {xiv }}$ et la philosophie 'analytique' des autres projets est que le DES ne fournit ni une liste d'entrées lexicales ni une liste de constructions associées à ces entrées $^{\mathrm{xv}}$. Dans cette entreprise, la question explicitement soulevée par Stein $(2005: 38-44)$ de l'articulation entre niveau conceptuel et niveau sémantique (qui le conduit à écarter pour son projet le terme d' " ontologie » au profit de " hiérarchie conceptuelle ») ne se pose pas : le matériau constitutif du réseau est uniquement constitué d'items lexicaux, sans intervention d'aucune catégorisation conceptuelle. De ce point de vue, le fondement du dictionnaire relève d'un pur structuralisme sémantique ${ }^{\mathrm{xvi}}$. Indépendamment de ce préalable qu'on peut questionner, deux des problèmes majeurs que doit affronter le DES sont d'une part le nombre très variable d'un domaine à l'autre des liens synonymiques disponibles (problème que ne connaît pas une approche à base conceptuelle qui crèe le nombre de niveaux hiérarchiques nécessaire pour la discrimination des sens et indirectement des items qui les véhiculent) et d'autre part le fait que dans de nombreux cas la relation de synonymie n'affecte pas seulement des items simples mais aussi des polylexèmes (figés) ou des phrasèmes (plus ou moins figés). Ce second problème est commun aux deux approches, analytique et émergentiste. 


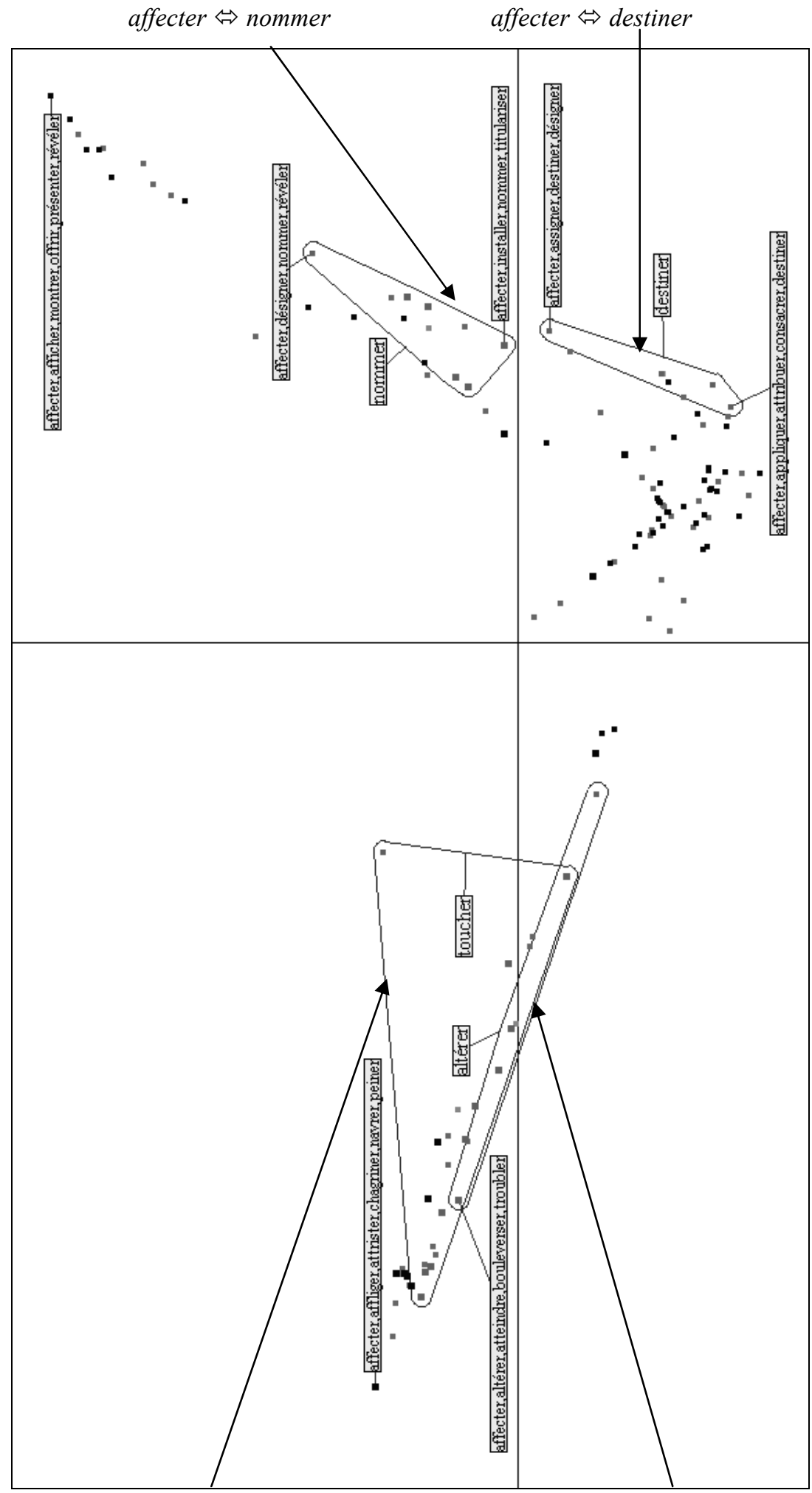

affecter $\Leftrightarrow$ toucher

affecter $\Leftrightarrow$ altérer

Figure 4: Projection de l'espace sémantique du verbe affecter sur le plan 1x2 


\section{Approche diachronique de la polysémie verbale}

\subsection{Le destin des rôles sémantiques à travers l'histoire des langues}

Je ferai référence ici aux travaux de P. Koch qui ont la particularité de combiner depuis de nombreuses années un examen minutieux de l'interface entre syntaxe et sémantique verbale dans les langues romanes (cf. Koch 1981) et l'élaboration d'une théorie cognitive du changement sémantique (cf. Blank 2000, Koch 2000, 2002, Gevaudan 2007). Ces deux orientations convergent dans différentes publications, en particulier Koch (2004). Je ne résumerai ici que la section 2 sur le jeu des transformations en diachronie entre les cadres conceptuels, les participants et la structure actancielle.

Pour Koch (2004:423) « tout changement diachronique concernant d'une quelconque manière les rôles des participants sont un composant intégral (et typique) du changement sémantique verbal ». Je reviendrai au $\S$ suivant sur un aspect de cette thèse sujet à débat.

Dans cette optique le premier type de changement évoqué est un changement «indépendant des places actancielles ». Par ex. dans (1)

\section{Nos amis [agent] ont bien dîné}

il s'est produit entre l'ancien français et le français moderne un simple changement de référence temporelle du midi au soir. La sémantique actancielle n'est pas en cause dans ce premier type.

Le second type concerne le «changement de perspective du cadre [conceptuel] relativement aux participants ». Tout verbe a un profil Thème-Rhème inhérent qui peut varier à travers les siècles, ex. (2) où les rôles de stimulus ${ }^{\text {xvii }}$ et d'expérient se sont intervertis :

$$
\begin{aligned}
& \text { Ces médicaments [stimulus] répugnent aux malades [expérient ] } \\
& \rightarrow \text { Ces malades [expérient] répugnent aux médicaments [stimulus] }
\end{aligned}
$$

Le troisième type touche le «changement des propriétés actancielles des participants », ex. (3) où le verbe source latin capere exprime une relation entre un agent-possesseur (final) et une possession, tandis que le verbe cible italien capire exprime une relation expérient-stimulus.

(3) LatClass. Marcus[agent-possesseur] capit baculum [possession]

$\rightarrow \quad$ It. Giovanni [expérient] capisce la mia riposta [stimulus]

Le quatrième type correspond à ceux des verbes réversibles dont la variante syntaxique intransitive est première, ex. (4) où le cadre primaire ne comporte qu'un patient réalisé comme sujet grammatical et le cadre dérivé un agent réalisé comme sujet et un patient réalisé comme objet direct. Koch (2005:24sq) propose un aperçu typologique des modes de réalisation de l'opposition Causatif vs. Récessif.

(4) The stone[patient] dropped

$$
\rightarrow \quad J o h n[\text { agent] dropped the stone[patient] }
$$

Enfin le cinquième type est l'inverse du quatrième avec la délétion d'un participant et de son rôle. Koch (p.426-7) en donne deux exemples. Le premier (5) illustre une évolution entre le latin classique et le français, le verbe latin pascere étant causatif avec un sujet agent, tandis que le verbe français paître qui en dérive est intransitif, ce qui suppose la délétion du référent de l'agent (à savoir le pâtre).

(5) LatClass. Marcus [agent] pascit oves [non-agent]

$\rightarrow \quad$ Fr. Les moutons [non-agent] paissent dans le pré [locatif circonstanciel]

Le second exemple met en jeu une redistribution plus complexe des rôles (6) : dans un premier temps le verbe louer (toujours quantitativement trivalent) prend comme sujet un " agent-amissif » (c'est-à-dire qui se dessaisit d'un bien) et comme oblique introduit par un à «acceptif» (destinataire du bien), dans un second temps le référent du sujet reste un agent mais complémentairement «acceptif » tandis que le rôle « amissif » passe à l'oblique, toujours introduit par $\grave{a}$. 
(6) La société immobilière [agent-amissif] a loué cet appartement [possédé] à un étudiant [acceptif] $\rightarrow \quad$ L'étudiant [agent-acceptif] a loué cet appartement [possédé] à une société immobilière [amissif]

Finalement, Koch classe ces cinq types dans les catégories classiques des changements de sens depuis Bréal (1897). Le type I (diner) relève d'une « métonymie indépendante des places actancielles », les types II, III et V de métonymies liées aux relations de contiguïté entre les participants (par ex. la redistribution des actants en II) et entre ceux-ci et le cadre prédicatif (par ex. l'accueil d'un actant en IV vs. le rejet d'un actant en V). Seul le type III (lat. capere $\rightarrow$ it. capire) illustre le processus de dérivation métaphorique. Les processus restants de généralisation et de spécialisation ne sont pas en cause ici.

La principale qualité de mode d'argumentation est sans doute sa systématicité qui permet de prendre en compte tous les types de changements, non seulement sémantiques, mais aussi dérivationnels et phraséologiques, comme le montre l'exemple (p.430) de la relation entre le cadre prédicatif d'OUVRIR et celui de S'OUVRIR (le cadre non causatif étant toujours dérivé dans les langues citées) : la dérivation s'effectue par changement de sens (c'est-à-dire par un verbe réversible) en anglais et grec moderne, par diathèse en français, roumain, russe et allemand, par flexion (ablaut) en hindi, par alternance vocalique (transfixe) en arabe et hébreu, par suffixation en oudmourte (roupe finno-ougrien), finnois, turc et souahéli, et par préfixation en lituanien.

Cependant le cas des verbes réversibles (type 4) pose un problème particulier qui sera abordé au §3.2, celui du domaine d'application de la propriété [+/-causatif]. Contrairement à la dynamicité, la transitionalité, la momentanéité, la relationalité ou l'agentivité, qui sont des propriétés (aspectuelles pour les premières et participative pour les deux dernières) du type de procès, la causativité est une propriété de la prédication verbale qui introduit un point de vue sur l'arrière-plan causal du type de procès exprimé (cf. François 2001, 2002) et l'opposition [+/-causatif] peut être marquée par un "changement de sens ", ex. La branche casse (sous l'effet de la tornade) $\rightarrow$ La tornade casse la branche, ou par une « diathèse récessive à marquant réfléchi » (terminologie de L. Tesnière), ex. La tornade brise la branche $\rightarrow$ La branche se brise (sous l'effet de la tornade).

\subsection{Les variations événementielles et la diversification des sens verbaux en diachronie}

\subsubsection{La double hypothèse à tester}

Nous achèverons ce tour d'horizon en proposant une double hypothèse sur l'émergence de l'effet de polysémie en matière de prédications verbales.

i. Une prédication verbale - définie comme une paire \{prédicat verbal, cadre prédicatif\}, cf. François 2003 - exprime un type de procès, si elle n'est pas instanciée (en termes de temps, d'aspect et de référence des arguments), ou un procès particulier, si elle est instanciée. Le procès prédiqué présente des propriétés de trois ordres : aspectuel, participatif et conceptuel.

ii. Une variation événementielle élémentaire n'affectant qu'une propriété aspectuelle ou participative n'entraîne pas à elle seule un d'effet de polysémie, mais seulement des 'variantes' (nous revenons cidessous sur cette notion chez Dubois \& Dubois-Charlier) incluant un changement de point de vue ou en termes plus techniques de 'structure informationnelle' (cf. Lambrecht, 1994). En revanche une variation conceptuelle et/ou un cumul de variations élémentaires d'ordre aspectuel ou participatif produisent un effet de polysémie.

A titre d'exemple de variation aspectuelle, le jeu des localisations temporelles dans la phrase

Hier Jean a déjeuné (a) à midi (b) en 1/2 heure (c) pendant des heures

produit trois variantes aspectuelles sans effet de polysémie, c'est-à-dire sans variation sur le type d'action accomplie par Jean. Dans la variante (a), la durée du déjeuner n'est pas prise en compte : midi repère le début de l'action (variante inchoative), dans la variante (b) le déjeuner est représenté comme un 
'accomplissement' (dans la terminologie de Z. Vendler, 1967), c'est-à-dire un procès télique. Ces deux variantes sont composables : Hier Jean a déjeuné à midi en $1 / 2$ heure, car le repérage de l'instant initial n'est pas incompatible avec l'indication d'une durée limitée. Dans la variante (c), le déjeuner est représenté comme une 'activité' sociale et/ou gastronomique et est incompatible avec le repérage de l'instant initial : *Hier Jean a déjeuné à midi pendant des heures.

Concernant la variation de propriétés participatives, considérons l'emploi transitif causatif du verbe entrer qui remonte à $1615^{\text {xviii }}$, mais seulement avec un objet non animé (ex. entrer le foin dans la grange). Récemment, cette restriction de sous-catégorisation a été levée avec des énoncés intégrant une métonymie tels que Je ne vous ai pas encore entré dans ma liste de destinataires. Cette variation participative limitée (la représentation du procès restant causative et agentive) n'est associée ni à une variation aspectuelle (le procès est toujours télique, et de ce fait dynamique et transitionnel) ni à une variation conceptuelle (il ne s'agit pas d'un emploi métaphorique). Il s'agit donc d'une simple variation constructionnelle ${ }^{\mathrm{xix}}$ sans effet de polysémie caractériséx

En revanche, quand, à partir du $17^{\mathrm{e}}$ siècle, le verbe demander accueille comme sujet une expression nominale non animée et éventuellement prédicative (variation participative, cf. Ce travail vous demandera une grande attention / La situation de crise demande une réaction énergique), une autre variation participative y est associée (le référent du sujet n'est plus un Agent), ainsi qu'une variation aspectuelle (un état relationnel se substitue à un événement) et surtout une variation conceptuelle (la prédication exprime une relation logique et non plus un acte de communication linguistique). La variation conceptuelle à elle seule produit un effet de polysémie, mais cet effet est conforté par les deux variations connexes, aspectuelle et participative.

\subsubsection{Les variantes de LVF : polytaxie sans polysémie}

Le point de vue défendu par P. Koch dans l'article examiné au §3.1 et étayé dans Koch (1991) et Waltereit (1998) à partir d'arguments historiques et dans Koch (2005) sur une base typologique, est qu'une variation d'arrangement des actants du type représenté par les verbes dits, selon les terminologies, réversibles, à renversement, labiles ou symétriques, est obligatoirement associé à une polysémie. Martin (1983) parle dans ce cas de polysémie 'sélectionnelle, c'est-à-dire produite par l'arrangement variable des actants sans variation du sémème verbal.

Inversement, sur les 25610 entrées des Verbes français, 66 présentent une double caractéristique intéressante : elles sont caractérisées à la fois par un schème composé de constructions du type A $\bullet \bullet \bullet$

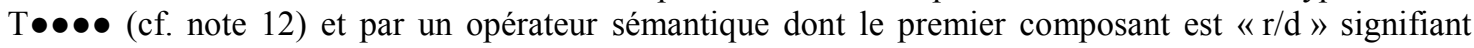
«rendre/devenir ». Il s'agit donc indiscutablement de 66 entrées du type réversible, lesquelles entrent dans trois schèmes composés: le premier A10 T3106 (construction intransitive à sujet humain; transitive à sujet humain et objet non animé, circonstant de 'modalité' étroitement lié) réunit 2 entrées, ex. mollir 03 (le sujet de la construction transitive correspond au circonstant privilégié de la construction intransitive: On mollit devant l'obstacle SOUS L'EFFET DE L'INACTION), le second A30 T1308 (construction intransitive à sujet non animé ; transitive à sujet humain et objet non animé, circonstant de moyen/instrument étroitement lié) en réunit 39, ex. baisser 04 (ex. avec circonstant privilégié : On baisse les prix / Les prix baissent AVEC DES MESURES ANTI-INFLATIONNISTES). Enfin le troisième A30 T3300 (construction intransitive à sujet non animé ; transitive à sujet et objet non animés, sans circonstant étroitement lié) en regroupe 25 , ex. raccourcir 04. 


\begin{tabular}{|l|l|l|l|l|l|l|l|}
\hline mollir 03 & PSY & T2b & $\begin{array}{l}\text { r/d mou-a } \\
\text { abs }\end{array}$ & céder,plier & $\begin{array}{l}\text { On m devant l'obstacle. } \\
\text { L'inaction a m leur résistance. }\end{array}$ & $\begin{array}{l}\text { A10 } \\
\text { T3106 }\end{array}$ & 2 entrées \\
\hline baisser 04 & MON & T4a & $\begin{array}{l}\text { r/d+qt bas } \\
\text { val }\end{array}$ & diminuer & $\begin{array}{l}\text { On b les prix,la pression. } \\
\text { Les prix,la pression b . }\end{array}$ & $\begin{array}{l}\text { A30 } \\
\text { T1308 }\end{array}$ & 39 entrées \\
\hline raccourcir 04 & MTO & T3a & r/d court tps & $\begin{array}{l}\text { diminuer } \\
\text { durée }\end{array}$ & $\begin{array}{l}\text { L'hiver r les jours. } \\
\text { Les jours,les nuits r . }\end{array}$ & $\begin{array}{l}\text { A30 } \\
\text { T3300 }\end{array}$ & 25 entrées \\
\hline
\end{tabular}

Du point de vue de la théorie des schèmes composés de constructions syntaxiques de Dubois \& DuboisCharlier ${ }^{\mathrm{xxi}}$ les constructions rassemblées dans un schème composé constituent des «variantes » transformationnelles ${ }^{\mathrm{xxi}}$, cf. p. 2 de l'édition papier :

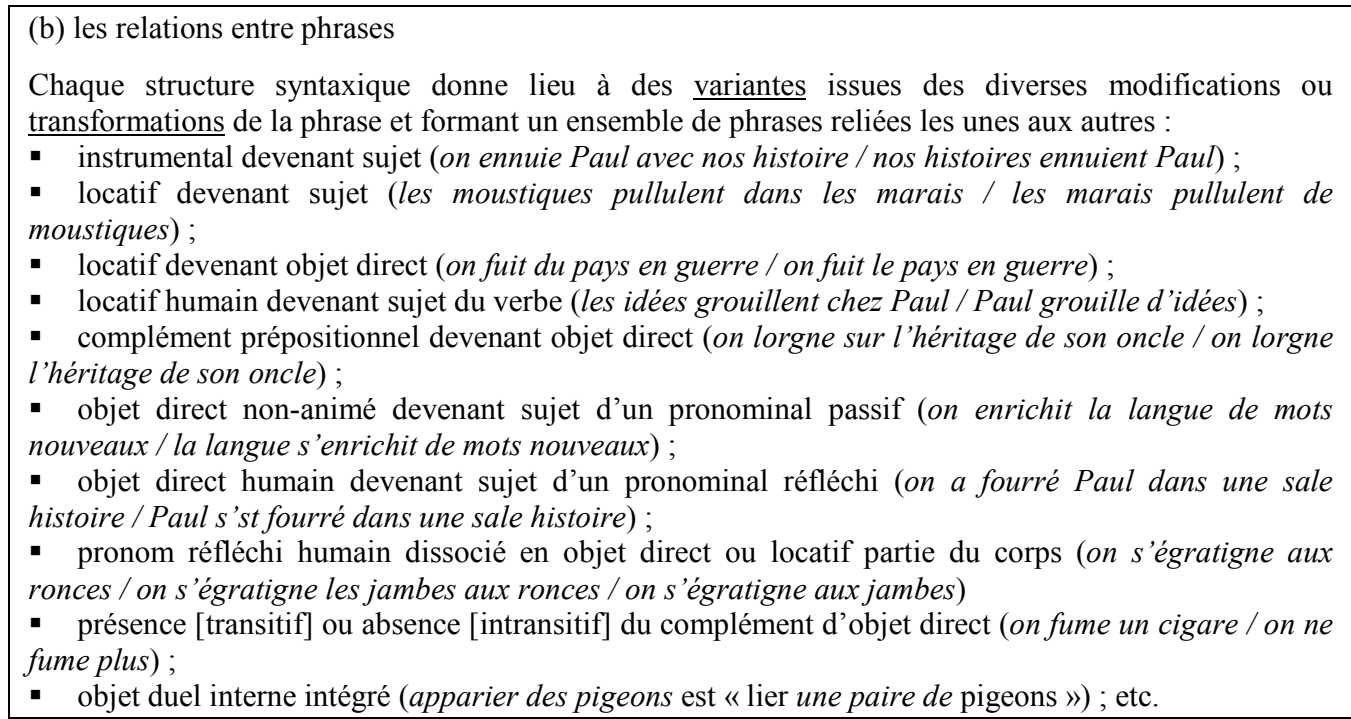

Ces deux points de vue sont donc apparemment incompatibles, mais on peut essayer de les accorder en considérant que les variations du sens de la prédication verbale issues d'une métonymie ou d'un réarrangement des actants entraînent un « effet de polysémie » faible, tandis que les variations affectant le noyau prédicatif (en général par métaphore, généralisation ou spécification) produisent un effet de polysémie fort.

\subsubsection{Les variations aspectuelles}

Les traits aspectuels primaires sont relatifs à la durée du procès (momentané vs. duratif) et à sa dynamicité (dynamique vs. statif) auxquels s'ajoutent pour les procès dynamiques la distinction entre téliques et atéliques ${ }^{\mathrm{xxiii}}$ (présence ou absence d'un changement) et pour les procès statifs celle entre permanents et transitoires (propriétés vs. états). Selon Givón (1989:59) un événement se définit, entre autres propriétés, comme un changement clairement visible ou saillant en termes de gradient de changement (rate of change over time), un événement prototypique est donc télique et ponctuel.

Les procès statifs (états transitoires et propriétés) s'opposent aux événements prototypiques et les procès téliques mais duratifs, les procès dynamiques atéliques (obligatoirement duratifs) et les états momentanés (ex. Marie sursauta à l'annonce de la nouvelle) sont autant de types marginaux d'événements.

Le verbe occuper présente une double illustration de variations aspectuelles élémentaires sans effet immédiat ${ }^{\mathrm{xxiv}}$ de polysémie. Le cadre syntaxique ne varie pas : qn:X occupe $q c<$ lieu $>: Y$. Le premier type de procès prédiqué est momentané \& télique (donc un événement prototypique), ensuite c'est la situation 
résultant de la prise de possession qui est prédiquée (événement ni momentané, ni télique), enfin c'est un état relationnel.

\subsubsection{Les variations participatives ${ }^{\mathrm{xxv}}$}

Ici aussi les variations élémentaires, par exemple les extensions et les réductions de valence ne produisent généralement pas d'effet de polysémie. Généralement les variations participatives doubles par dégradation $^{\text {xxvi }}$ de l'un des actants et promotion compensatoire d'un autre actant n'entraînent pas non plus de polysémie, tout au plus une variation de la structure informationnelle (cf. Levin \& Rappaport-Hovav 2005). Les verbes manquer et livrer fournissent deux illustrations de ce que Tesnière (1959) appelle « interversion double des actants » dans son chapitre sur la métataxe. Dans le premier cas, le premier et le tiers actant échangent leur place (avec un changement de proposition entre $\grave{a}$ et $d e$ ).

Dans le second cas, c'est le second et le tiers actant qui échangent leur place (également avec un changement de préposition entre $\grave{a}$ et $e n)$.

On pourrait faire valoir que les paraphrases changent, mais il est clair que nous sommes en présence d'équivalences par interversion :

\section{$\mathrm{X}$ fait défaut à $\mathrm{Y} \Leftrightarrow \mathrm{Y}$ est dénué de $\mathrm{X}$ \\ $\mathrm{X}$ apporte $\mathrm{Y}$ à $\mathrm{Z} \Leftrightarrow \mathrm{X}$ approvisionne $\mathrm{Z}$ en $\mathrm{Y}$}

En revanche, dans le cas d'une greffe entre un cadre prédicatif primaire et une construction (dénuée de lexème verbal) fonctionnant comme greffon (cf. François \& Sénéchal 2006), cette variation participative par attraction entraîne un effet de polysémie. Le verbe appeler en fournit un exemple. Dans le cadre prédicatif $\mathrm{X}<\mathrm{qn}>$ appelle $\mathrm{Y}<\mathrm{qn}>$, il prédique primairement une prise de contact par la voix. Puis il subit l'attraction d'une construction de requête $\mathrm{X}<\mathrm{qn}>\mathrm{V} \mathrm{Y}<\mathrm{qn}>\grave{a} \mathrm{Z}_{\mathrm{INF}}[=$ action de $\mathrm{Y}]$, où le contenu de l'infinitive exprime une action que $\mathrm{X}$ pousse $\mathrm{Y}$ à accomplir, si bien qu'il s'associe à ce nouveau cadre pour prédiquer un acte de requête.

\subsubsection{Les variations conceptuelles}

Nous avons évoqué au \$3.2.1 le cas, pour le verbe demander, de l'émergence au $17^{\mathrm{e}}$ siècle d'un sens de simple implication à partir de la valeur de requête (phénomène qui touche ultérieurement par analogie d'autres verbes de requête: requérir, exiger, réclamer et appeler, cf. François 2007b). Ce type de variation conceptuelle, qu'on retrouve également en dehors de la communication linguistique avec par exemple les verbes conduire, entraîner, mener ou pousser (ex. Le pâtre mène le troupeau au pâturage $\rightarrow$ L'alcool l'a mené à la folie) est analysable à l'aide de la notion de greffe (cf. François \& Sénéchal 2006 sur la base de Goldberg 1995). Sur une prédication primaire de requête [N1: qn $<\mathrm{x}>$ demande $_{\mathrm{V}} \mathrm{N}: \mathrm{qch}<\mathrm{y}>$ $\grave{a} \mathrm{~N} 3: \mathrm{qn}<\mathrm{z}>$ ] s'opère la greffe d'un cadre prédicatif dont le sujet réfère à une action $[\mathrm{N} 1: \mathrm{qch}<\mathrm{action}: \mathrm{a}>$

${ }_{\mathrm{v}} \mathrm{N} 2: \mathrm{q} c h<\mathrm{y}>(\grave{a} \mathrm{~N} 3: \mathrm{qn}<\mathrm{z}>)$ ], ce qui produit une prédication résultante de relation action-moyen, $\mathrm{cf}$. Tableau $3^{\text {xxvii }}$ 


\begin{tabular}{|c|c|c|c|c|}
\hline $\begin{array}{c}\text { prédication } \\
\text { source }\end{array}$ & $\begin{array}{c}\mathrm{N} 1: \mathrm{qn}<\mathrm{x}> \\
\text { Marie }\end{array}$ & demande & $\begin{array}{c}\mathrm{N} 2: \mathrm{qch}<\mathrm{y}> \\
\text { de la patience }\end{array}$ & $\begin{array}{c}\text { à } \mathrm{N} 3: \mathrm{qn}<\mathrm{z}> \\
\text { aux élèves }\end{array}$ \\
\hline $\begin{array}{c}\text { cadre } \\
\text { prédicatif } \\
\text { greffon }\end{array}$ & $\mathrm{a}:$ action & $\begin{array}{c}\mathrm{V} \\
\text { nécessite, } \\
\text { implique\} }\end{array}$ & $\mathrm{qch}$ & $\begin{array}{c}\text { (de la part } \\
\text { de } \mathrm{N})\end{array}$ \\
\hline $\begin{array}{c}\text { prédication } \\
\text { résultante }\end{array}$ & $\begin{array}{c}\mathrm{N} 1: \mathrm{qch}<\mathrm{act}: \mathrm{a}> \\
\text { La résolution du } \\
\text { problème }\end{array}$ & demande & de la patience & $\begin{array}{c}\text { (à } \mathrm{N} 3: \mathrm{qn}<\mathrm{z}> \\
\text { aux élèves }\end{array}$ \\
\hline
\end{tabular}

Tableau 3 : Genèse par greffe d'une prédication de relation action-moyen à l'aide du prédicat verbal demander

\subsubsection{La perte de prédicativité}

Enfin, en empruntant une métaphore à l'astrophysique, on peut dire que les prédications verbales sont comparables à des astres qui passent par différents états physiques, l'aboutissement ultime de ces changements d'état étant la métamorphose en trou noir. Le 'trou noir' des variations susceptibles d'affecter une prédication verbale, c'est la perte de prédicativité ${ }^{\text {xxviii }}$. Elle peut constituer un destin plénier, c'est le cas en français contemporain par exemple pour daigner ou s'abstenir qui ont perdu tout emploi prédicatif et prochainement pour faillir qui risque de perdre ses derniers emplois prédicatifs (ex. Paul a failli à tous ses devoirs, Marie a failli), ou seulement une voie de garage accessoire, comme pour manquer qui connaît deux types d'emploi comme semi-auxiliaire, ex.

Paul a manqué (de) créer un accident en passant au feu rouge $\approx$ faillir

Marie ne manquera pas d'être étonnée par cette nouvelle $\approx$ Immanquablement, Marie sera étonnée par cette nouvelle.

\section{Bilan}

\subsection{En synchronie}

L'approche de la polysémie (entre autres) verbale chez les linguistes français est dominée, malgré les travaux d'inspiration structuraliste de R. Martin ou F. Rastier, par la recherche d'un 'signifié de puissance' (J. Picoche) ou d'une 'forme schématique' (J.J. Franckel, D. Paillard, B. Victorri entre autres), c'est-à-dire par une conception 'verticale' de la polysémie. L'option des chercheurs en lexicographie électronique consiste en revanche à dégrouper largement le champ sémasiologique de chaque lexie avant d'examiner le degré de proximité entre les entrées lexicales ainsi constituées. C'est vrai pour Stein (2005), pour le Dictionnaire intégral de Mémodata comme pour Les verbes français. Selon les bases de données lexicographiques, le poids respectif des deux classements corrélés, le conceptuel et le syntaxique, peut varier. Le Dictionnaire électronique des synonymes du CRISCO (cf. François 2007a) occupe une position particulière, car ce sont les cliques de synonymes qui se substituent aux entrées lexicales.

\subsection{En diachronie}

Toutes classes lexicales confondues, les travaux les plus détaillés et les mieux assurés en théorie sont ceux de l'école allemande autour de P. Koch et A. Blank. Du point de vue de la polysémie verbale, P. Koch défend l'idée que l'essentiel des variations syntaxiques représente des cas de polysémie. Dans l'approche transformationnaliste de Dubois \& Dubois-Charlier (1997a,b) au contraire, certaines variations sont considérées comme de simples alternances, c'est-à-dire des cas de polytaxie sans polysémie. En tout état de cause, on peut distinguer quatre facteurs cumulables de diversification de sens : les variations aspectuelles, participatives et conceptuelles, ainsi que la perte de prédicativité (assimilable à une perte de valeur conceptuelle, éventuellement combinée à une perte de valeur participative et/ou aspectuelle). 


\section{Références}

Ben Salah-Tlili, I. (2007), "Contribution à l'étude des 'verbes symétriques' en français contemporain », in J. François \& A. Brahim (dir.), Sémantique et morphosyntaxe du verbe, Cahier n`23 du CRISCO, Université de Caen \& CNRS, p.15-37.

Blake, B. (1991), Relational Grammar. London : Routledge \& Kegan.

Blank, A. (1997), Prinzipien des lexikalischen Bedeutungswandels am Beispiel der romanischen Sprachen. Beihefte zur Zeitschrift für Romanische Philologie.Tübingen : Niemeyer.

Blank, A. (2000), « Pour une approche cognitive du changement sémantique : aspect sémasiologique ». In J. François (dir.) p. 59-74.

Blank, A. (2001), Einführung in die lexikalische Semantik für Romanisten. Tübingen : Niemeyer.

Bréal, M. (1897/2005), Essai de sémantique (Science des significations). $3^{\mathrm{e}}$ édition de 2005 reproduisant la $2^{\mathrm{e}}$ de 1897. Limoges : Lambert-Lucas

Busse, W. (1974), Klasse - Transitivität-Valenz. Munich : Fink.

Darmesteter, A. (1887/1979), La vie des mots étudiée dans leurs significations, Paris, Delagrave [1887], Champ Libre [1979].

Desclés, J.P. (2005), «Polysémie verbale, un exemple: le verbe 'avancer'». In: O. Soutet (dir. 2005), La polysémie. Paris : Presses de l’Université de Paris-Sorbonne, p.111-136.

Dubois, J. (1969), Grammaire structurale du farnçais - Tome 3 : La phrase et les transformations. Paris : Larousse.

Dubois, J. \& Dubois-Charlier, F. (1997a), Les verbes français. Paris : Larousse

Dubois, J. \& Dubois-Charlier, F. (1997b) : «Synonymie syntaxique et classsification des verbes français », Langages, 128, 51-71.

Dutoit, D. \& J. François (2007). « Changer et ses synonymes majeurs entre syntaxe et sémantique : le classement des verbes français en perspective ». Langue Française 153, p.40-57.

Fillmore, Ch. \& S. Atkins, S. (2000), «Describing polysemy : the case of 'crawl'». In : Y. Ravin \& C. Leacock (eds, 2000), Polysemy - Theoretical and computational approaches. Oxford : Oxford University Press, p. 91-110

Franckel, J.J., Paillard, D. \& Saunier, E. (1997) « Modes de régulation et la variation sémantique d'une unité lexicale. Le cas du verbe passer ». In : P. Fiala, P. Lafon \& M.F. Piguet (dir. 1997), La locution : entre lexique, syntaxe et pragmatique - Identification en corpus, traitement, apprentissage. Paris : Klincksieck, p.49-68.

François, J. (1989), Changement, Causation, Action - Trois catégories sémantiques fondamentales du lexique verbal français et allemand. Genève : Droz.

François, J. (dir. 2000), Théories contemporaines du changement sémantique. Mémoire I, Nouvelle série. Société de Linguistique de Paris. Louvain : Peeters

François, J. (2001) «L'arrière-plan causal dans la mise en discours du changement d'état (présentation d'un test sur les verbes psychologiques en français) » In : A. Rousseau (dir.), La sémantique des relations. Edition du Conseil Scientifique de l'Université Charles-de-Gaulle - Lille 3, p.135-150

François, J. (2002): «La catégorisation verbale » In F. Cordier \& J. François (dir.), Catégorisation et langage. Paris : Hermès Lavoisier, p.53-82.

François, J. (2006), «Le continuum de transitivité en français et la dimension universelle de la 'participation' ». Zeitschrift für französische Sprache und Literatur 90-1, p.1-20.

François, J. (2007a), Pour une cartographie de la polysémie verbale. Bibliothèque de la Société de Linguistique de Paris $n^{\circ}$ 92. Louvain : Peeters

François, J. (2007b), «L'émergence d'une construction : comment plusieurs verbes français de requête ont acquis l'aptitude à exprimer l'implication à partir du $17^{\mathrm{e}}$ siècle ». BSL CII, volume 1. pp.193-215.

François, J. (2008a), Une approche diachronique quantitative de la polysémie verbale. Cahier 24 du CRISCO [téléchargeable sur le site www.crisco.unicaen.fr]. 
François, J. (2008b) «The French verb affecter in 3 types of news articles: A geometric representation with semantactic areas ». Actes du colloque JADT 2008.

François, J. (2008c), «Entre événements et actions: les schèmes composés de constructions syntaxiques du dictionnaire Les verbes français de J. Dubois \& F. Dubois-Charlier ». In I. Novakova \& Z. Guentcheva (dir.), Syntaxe et sémantique des prédicats. Lidil 37

François, J. (à par.) «Perte de prédicativité et auxiliarisation en français : Examen intégratif de deux ressources lexicales ». In : A. Ibrahim (dir.), Prédicats, prédications et structures prédicatives. [Editeur non spécifié]

Gevaudan, P. (2007), Typologie des lexikalischen Wandels - Bedeutungswandel, Wortbildung und Entlehnung am Beispiel der romanischen Sprachen. Tübingen : Stauffenburg.

Grimshaw, J. (1990), Argument structure. Cambridge (Mass.) : M.I.T. Press

Guilbert, L. (1975), La créativité lexicale. Paris : Larousse

Kleiber, G. (1999), Problèmes de sémantique - La polysémie en question. Villeneuve d'Ascq: Presses du Septentrion.

Klepousniotou, E. (2007), «Reconciling linguistics and psycholinguistics : On the psychological reality of linguistic polysemy ». In Rakova, M., Pethö, G \& Rákosi, C (eds.): 17-46.

Koch, P. (1991), «Semantische Valenz, Polysemie und Bedeutungswandel bei romanischen Verben », in: Koch. P./Krefeld, Th. (édd.), Connexiones Romanicae. Dependenz und Valenz in romanischen Sprachen, Tübingen: Niemeyer 1991, 279-306 en part. 294s.;

Koch, P. (2000), «Pour une approche cognitive du changement sémantique: aspect onomasiologique ». In J. François (dir.) p. 75-96.

Koch, P. (2002), «Il ne me faut plus nule rien. »-Changement sémantique , métataxe et réanalyse. In P. Blumenthal \& P. Koch (dir.) Valence : perspectives allemandes. Syntaxe \& Sémantique 4, p.67-108.

Koch, P. (2004), «Rollensemantik - diachronische Aspekte », in: Kailuweit, R./Hummel, M. (édd.), Semantische Rollen, Tübingen: Narr, p.421-434

Koch, P. (2005), « Aspects cognitifs d'une typologie lexicale synchronique. Les hiérarchies conceptuelles en français et dans d'autres langues », Langue française 145, p.11-33

Krötsch, M. \& Österreicher, W. (2002), «Dynamique des configurations actancielles: Modifications des constructions verbales en français non standard ». In : P. Blumenthal \& P. Koch (dir.), Valence : perspectives allemandes. Syntaxe \& Sémantique 4, Presses Universitaires de Caen, p.109-137.

Lambrecht, K. (1994), Information structure and sentence form. Cambridge (GB) : Cambridge University Press.

Levin, B \& Rappaport-Hovav, M (2005), Argument realization. Oxford et al. : Oxford University Press.

Martin, R. (1983), Pour une logique du sens. Paris : PUF

Picoche, J. (1986), Les structures sémantiques du lexique français. Paris : Nathan-Université.

Picoche, J. \& Rolland, J.C. (2002), Dictionnaire du français usuel. Bruxelles : De Boeck-Duculot

Picoche, J. (1994), « A 'continuous definition' of polysemous items: its basis, resources and limits ». In : C. Fuchs \& B. Victorri (eds. 1994), Continuity in linguistic semantics. Amsterdam : Benjamins. p.77-92.

Ploux, S. \& H. Ji (2003), «A model for matching semantic maps between languages (French-English, EnglishFrench ». Computational Linguistics 29-2, p.155-178.

Ploux, S. \& Victorri, B. (1998,) «Construction d'espaces sémantiques à l'aide de dictionnaires de synonymie », TAL $39: 161-182$.

Pustejovksy, J. (1995), The generative lexicon. Cambridge (Mass.) : MIT-Press.

Rakova, M., Pethö, G \& Rákosi, C (eds.), The cognitive basis of polysemy - New sources of evidence for theories of word meaning,. Frankfurt am Main et al. : Peter Lang.,

Rastier, F. (1991), Sémantique et recherches cognitives. Paris : PUF. 
Rousseau, A. (2000), «L'évolution lexico-sémantique : explications traditionnelles et propositions nouvelles ». In J. François (dir.), p.11-30

Stein, A. (2005), Semantische Repräsentation italienischer Verben. Linguistische Arbeiten 499 ? Tübingen: Niemeyer

Tesnière, L. (1959), Eléments de syntaxe structurale. Paris : Klincksieck.

van Valin, R. (2005), Exploring the syntax-semantics-interface. Cambridge: Cambridge University Press.

Vendler, Z. (1967), Linguistics in philosophy, Chapter 4, «Verbs and times », p.97-121, New-York, Cornell University Press.

Victorri, B. \& Fuchs, C. (1996), La polysémie - Construction dynamique du sens. Paris : Hermès.

Waltereit, R. (1998), Metonymie und Grammatik. Kontiguitätsphänomene in der französischen Satzsemantik, Tübingen: Niemeyer 1998, 84-88

\footnotetext{
${ }^{\text {i }}$ Dans la terminologie de la Functional Grammar de S. Dik (1997), équivalent à la structure argumentale de Grimshaw (1990).

ii [Picoche, 1986] pour l'étude succincte de nombreux verbes, [Picoche 1994] pour l'étude approfondie de marcher et [Picoche \& Rolland, 2002] pour un aboutissement dictionnairique.

iii «Sur la ligne du temps historique, cette situation [la monosémie] peut être parfaitement durable, ou, un beau jour, se modifier sous l'effet d'une figure de style, métaphore ou métonymie, qui vient à se lexicaliser, ou de quelque processus d'abstraction » [Picoche 1986, p.73]. Sur les différents processus en cause, cf. [Blank 1997, 2000], [Koch 2000] et le chapitre 3 'Semantische Filiation und Bedeutungswandel' (Filiation sémantique et changement de signification) de [Gevaudan 2007]. Pour une introduction générale à la sémantique historique cognitive, cf. (Blank 2001 : Chapitre 4] et pour une évaluation comparative des approches anciennes et contemporaines voir [Rousseau 2000].

${ }^{\text {iv }}$ Sous-titre de l'article de E. Klepousniotou (2007) dans ce volume.

$\checkmark$ Le diagramme est produit par le programme IV (Italian Verbs) et la pertinence des regroupements d'entrées lexicales obtenu est évalué par le programme SIC (Semantic Interpretation in Context), cf. Stein (2005, Chapitre 7 «Dokumentation der Daten und Programme »)

${ }^{v i}$ J. Dubois a dirigé la publication entre autres du Dictionnaire du Français Contemporain (1966) et du LEXIS de la langue française (1975) chez Larousse.

${ }^{v i i}$ L'accessibilité des deux fichiers, l'édition papier désormais épuisée en format image et l'édition électronique en format tableur sur le site du laboratoire MoDyCo [http://www.modyco.fr/?Ressources] permet de mesurer les avantages des deux formes d'édition.

${ }^{\text {viii } L e s ~ c h a m p s ~ q u i ~ n ' i n t e r v i e n n e n t ~ p a s ~ d a n s ~ l a ~ p r e ́ s e n t a t i o n ~(c l a s s e s ~ d ~ c o n j u g a i s o n ~ e t ~ d e ~ d e ́ r i v a t i o n, ~ e t c .) ~ o n t ~ e ́ t e ́ ~}$ écartés. Abréviations : Dom(aine d'usage), Css : classe syntactico-sémantique, Constr(uction).

${ }^{\text {ix }}$ Pour plus de détails, voir Dutoit \& François (2007) qui propose p.45 un regroupement identique au final à celui du tableau 2 sur la base des classes génériques: les 18 entrées comportant dans la rubrique Opérateur «mut» ou « li.mut » relèvent de la classe $\mathrm{T}$ (transformations), tandis que les 3 entrées restantes relèvent de la classe $\mathrm{E}$ : (faire) sortir/venir, $\mathrm{N}$ : (dé)munir qn de qc) ou $\mathrm{R}$ : créer, fabriquer, mettre dans un état.

${ }^{x}$ Trois classes génériques sont représentées : $\mathrm{N}:$ : (dé)munir qn de qc », R : «mettre en état le corps de qn » et $\mathrm{T}$ : «(se) transformer». Respectant les principes du classement lexicographique traditionnel, les classes syntacticosémantiques se basent sur la sous-catégorisation des actants et sur l'opposition sémantique entre emploi propre et emploi figuré.

${ }^{x i}$ Les exemples ne présentent pas toujours les différentes constructions constituant le schème de constructions composé. Ainsi pour changer 06, il faudrait un exemple pour la construction pronominale P3000 (sujet non animé sans autres contraintes), tel que Le sens se change avec une virgule.

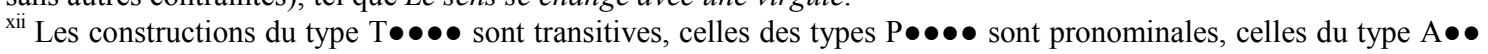
sont intransitives et celles du type $\mathrm{N} \bullet \bullet$ sont transitives indirectes. Pour tous les types, le $1^{\mathrm{er}}$ indicateur concerne la sous-catégorisation du sujet (1: humain, $2:$ animal, $3:$ non animé, $9:+/$-animé, etc.). Pour les types Tet $\mathrm{P}$ le $2^{\mathrm{e}}$ indicateur concerne celle de l'objet direct, le $3^{\mathrm{e}}$ le régime prépositionnel de l'objet second s'il y en a un (ex. a : $a, \mathrm{c}:$ avec; $\mathrm{k}$ : pour) et le $4^{\mathrm{e}}$ éventuellement un type de circonstant étroitement lié au sémantisme de l'entrée verbale (ex. changer 06, T1306: modalité). Pour les entrées du type A, le $2^{\mathrm{e}}$ indicateur concerne un circonstant étroitement lié à l'entrée (comme le $4^{\mathrm{e}}$ pour les types $\mathrm{T}$ et $\mathrm{P}$ ), pour celles du type $\mathrm{N}$, le $2^{\mathrm{e}}$ indicateur concerne le régime prépositionnel
} 
du complément indirect (cf. Dubois \& Dubois-Charlier 1997, p.7-8 et François, Le Pesant \& Leeman 2007, §3.1, Tableaux 4 à 7 ).

xiii Les visualisations correspondant à ces quatre aires sémantaxiques sont présentées dans François (2008b).

xiv Le calcul des interdistances est effectué par le logiciel VisuSyn élaboré par Bernard Victorri et Sabine Ploux et développé par Jean-Luc Manguin.

xv Le DES est syntaxiquement "aveugle », les données syntaxiques des 7 dictionnaires source ayant été volontairement mises de côté dans l'entreprise de mutualisation et de symétrisation des liens synonymiques qui constituent le réseau sémantique.

${ }^{x v i}$ Ce caractère structuraliste de l'entreprise n'a pas empêché Ploux \& Ji (2003), exploitant un dictionnaire électronique des synonymes français développé à l'Institut des Sciences Cognitives de Lyon (http://dico.isc.cnrs.fr), de même origine mais indépendant du DES, de tenter la visualisation d'un espace sémantique bilingue, constitué de l'espace sémantique d'un item lexical d'une L1 à partir de ses synonymes en L1 et des traductions de ses synonymes en L2, ces items de L2 étant eux-mêmes sémantiquement interconnectés. Les cliques réultant de la mutualisation des deux ressources peuvent comporter des synonymes des deux langues ou seulement de l'une des deux. De ce fait le sous-espace de chaque item de L2 apte à traduire l'item examiné de L1 dans un contexte particulier est repérable sur la visualisation.

xvii « Stimulus » traduit l'anglais « experienced».

xviii Datation empruntée à la rubrique HISTOIRE de l'article entrer du Trésor de la Langue Française.

xix Variation qui profite sans doute de l'accroissement du nombre des verbes 'symétriques' ou 'réversibles', cf. les grandes surfaces explosent les prix. La variation inverse décausative, touchant particulièrement les verbes expérienciels (ex. je fatigue, j'angoisse, j'hallucine) ne s'accompgne pas non plus d'un effet de polysémie (sur ces types de variation de valence, cf. Krötsch \& Österreicher 2002, François 2006, Ben Salah-Tlili 2007).

${ }^{x x}$ L'expression «sans effet de polysémie » est sujette à débat, comme me le fait remarquer Peter Koch avec de bons arguments, voir le $\$ 3.2 .2$ ci-dessous.

${ }^{x x i}$ Cette théorie remonte au $3^{\mathrm{e}}$ tome de la Grammaire structurale du français de J. Dubois, sous-titré «La phrase et les transformations », 1969

xxii On sait que toute une école de linguistique française, représentée en particulier, outre les travaux de J. Dubois, par les collaborateurs du LADL et ceux du LDI, reste fidèle à la conception des transformations développée originellement par Z. Harris et par M. Gross. Dans cette conception, certaines transformations préservent le noyau prédicatif, ce sont celles que Dubois \& Dubois-Charlier présentent comme des « variantes ».

xxiii Je ferai abstraction de la distinction complémentaire entre transitionnel et non transitionnel (cf. François 1989, Chapitre IV).

xxiv Comme la prévoit la variante 'polysémie par enchaînement' de Darmesteter (1887) et sa reformulation par Wittgenstein comme 'ressemblance de famille', l'enchaînement des deux variations produit finalement un effet de polysémie.

${ }_{\mathrm{xxv}}$ Par convention, 1 symbolise le prime actant (l'Agent dans une proposition syntaxiquement et sémantiquement transitive), 2 le second actant (le Patient dans ce type de proposition), 3 l'actant oblique et $\mathrm{C}$ les circonstants.

${ }^{\text {xxvi }}$ La notion de « dégradation » (angl. demotion) s'applique, dans la terminologie issue de la Relational Grammar (cf. Blake 1991) à l'une des variations $1 \rightarrow 2,1 \rightarrow 3,2 \rightarrow 3,2 \mid 3 \rightarrow \mathrm{C}$, les variations inverses étant désignées comme « promotions ».

xxvii Il est à noter que dans la prédication résultante de relation action-moyen, demander conserve de son cadre prédicatif source la possibilité d'introduire un complément prépositionnel introduit par à, alors que les verbes typiques de relation action-moyen, nécessiter et impliquer ne sont compatibles qu'avec un circonstant du type de la part de $\mathrm{N}$ : qn.

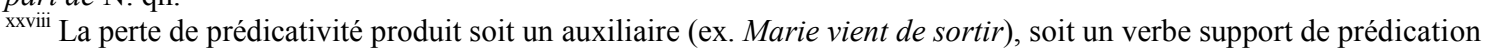
nominale (ex. Le prêtre donne l'absolution), soit une tête de locution verbale (ex. Jean prend part à la manifestation). Pour plus de détails, voir François (à paraître). 